\title{
Thulium fiber laser recanalization of occluded ventricular catheters in an ex vivo tissue model
}

\author{
Thomas C. Hutchens \\ David A. Gonzalez \\ Luke A. Hardy \\ C. Scott McLanahan \\ Nathaniel M. Fried
}




\title{
Thulium fiber laser recanalization of occluded ventricular catheters in an ex vivo tissue model
}

\author{
Thomas C. Hutchens, ${ }^{a}$ David A. Gonzalez, ${ }^{a}$ Luke A. Hardy, ${ }^{a}$ C. Scott McLanahan, ${ }^{b}$ and Nathaniel M. Fried ${ }^{a, *}$ \\ aUniversity of North Carolina at Charlotte, Department of Physics and Optical Science, North Carolina, United States \\ ${ }^{b}$ Carolinas Medical Center, Adult Hydrocephalus Specialty Clinic, Charlotte, North Carolina, United States
}

\begin{abstract}
Hydrocephalus is a chronic medical condition that occurs in individuals who are unable to reabsorb cerebrospinal fluid (CSF) created within the ventricles of the brain. Treatment requires excess CSF to be diverted from the ventricles to another part of the body, where it can be returned to the vascular system via a shunt system beginning with a catheter within the ventricle. Catheter failures due to occlusion by brain tissues commonly occur and require surgical replacement of the catheter. In this preliminary study, minimally invasive clearance of occlusions is explored using an experimental thulium fiber laser (TFL), with comparison to a conventional holmium: yttrium aluminium garnet (YAG) laser. The TFL utilizes smaller optical fibers $(<200-\mu \mathrm{m}$ OD) compared with holmium laser $(>450-\mu \mathrm{m} \mathrm{OD}$ ), providing critical extra cross-sectional space within the 1.2-mm-inner-diameter ventricular catheter for simultaneous application of an endoscope for image guidance and a saline irrigation tube for visibility and safety. TFL ablation rates using $100-\mu \mathrm{m}$ core fiber, 33-mJ pulse energy, $500-\mu \mathrm{s}$ pulse duration, and $20-$ to $200-\mathrm{Hz}$ pulse rates were compared to holmium laser using a $270-\mu \mathrm{m}$ core fiber, $325-\mathrm{mJ}, 300-\mu \mathrm{s}$, and $10 \mathrm{~Hz}$. A tissue occluded catheter model was prepared using coagulated egg white within clear silicone tubing. An optimal TFL pulse rate of $50 \mathrm{~Hz}$ was determined, with an ablation rate of $150 \mu \mathrm{m} / \mathrm{s}$ and temperature rise outside the catheter of $\sim 10^{\circ} \mathrm{C}$. High-speed camera images were used to explore the mechanism for removal of occlusions. Image guidance using a miniature, $0.7-\mathrm{mm}$ outer diameter, 10,000 pixel endoscope was explored to improve procedure safety. With further development, simultaneous application of TFL with small fibers, miniature endoscope for image guidance, and irrigation tube for removal of tissue debris may provide a safe, efficient, and minimally invasive method of clearing occluded catheters in the treatment of hydrocephalus. $\odot 2017$ Society of PhotoOptical Instrumentation Engineers (SPIE) [DOI: 10.1117/1.JBO.22.4.048001]
\end{abstract}

Keywords: ablation; hydrocephalus; holmium; laser; shunt; thulium; ventricular catheter.

Paper 170106R received Feb. 14, 2017; accepted for publication Apr. 7, 2017; published online Apr. 21, 2017.

\section{Introduction}

\subsection{Hydrocephalus and Shunt Revision Surgery}

Hydrocephalus is a chronic medical condition that occurs in individuals who, for a variety of reasons, are unable to reabsorb the cerebrospinal fluid (CSF) that is continuously created within the ventricles of the brain. While hydrocephalus is often associated with newborn babies, this condition also affects a large number of elderly adults, individuals suffering from dementia, and patients suffering from traumatic brain injuries. Overall, there are approximately 1 million Americans living with hydrocephalus. ${ }^{1}$ The primary treatment for most types of hydrocephalus requires the excess CSF to be diverted from the ventricles to another part of the body where it can be returned to the vascular system via a sometimes complex shunt system beginning with a catheter placed within the ventricle. This shunt system, first developed over 50 years ago, has one of the highest failure rates of any medical device. The most common complication is obstruction of the lumen and the 500- $\mu$ m-diameter ventricular holes at the distal tip of the proximal catheter due to parenchyma infiltration, choroid plexus tissue migration, and accumulation of inflammatory cells (e.g., macrophages and astrocytes). The surgical revision rate for CSF shunts is greater than $78 \%$ in children and about $33 \%$ in adults. ${ }^{2}$ The 1 -year shunt replacement rate is greater than $40 \% .^{3}$ The standard method for resolving obstruction or occlusion of the ventricular catheter is to surgically remove and replace it. This procedure takes about $2 \mathrm{~h}$, requires general anesthesia, results in surgical scars, requires a hospital stay of 1 day or more, and carries a significant risk of hemorrhage. ${ }^{4}$ The average cost of shunt revision surgery exceeds $\$ 35,000$ due in part to operating room and hospital recovery costs. Overall, medical costs for hydrocephalus are over $\$ 1$ billion per year. ${ }^{5}$

\subsection{Minimally Invasive Removal of Catheter Occlusions}

There is currently no accepted noninvasive or minimally invasive technique for clearance of obstructed ventricular catheters. A minimally invasive method would be ideal, preventing surgical complications, improving patient quality of life, and reducing operating room and recovery costs associated with surgery. Several studies have reported both experimental and clinical use of energy-based devices including electrocautery, ${ }^{6}$ ultrasound, ${ }^{7-9}$ and laser ${ }^{10,11}$ for prevention or clearance of obstructed catheters. However, these techniques have limitations. Electrocautery may produce excessive heat and unacceptable damage to the catheter and adjacent tissue. Ultrasound requires a large probe incorporating a distal transducer and is, therefore, 
not optimal for insertion through the small passages in current catheter designs. The holmium: yttrium aluminium garnet (YAG) laser, a common surgical laser, may also damage the inside of the catheter wall due to its high pulse energy operation. ${ }^{10,11}$ Furthermore, for at least some of these energybased techniques, real-time image guidance via endoscopy is not feasible, so confirmation of safe and complete clearance of the catheter cannot be visually confirmed.

\subsection{Thulium Fiber Laser}

Recent advancements in both fiber laser and endoscope technologies may enable minimally invasive recanalization of occluded ventricular catheters. Thulium fiber lasers (TFL) are capable of delivering sufficient power $(>10 \mathrm{~W})$ through small $(50$ - and $100-\mu \mathrm{m}$ core) optical fibers for tissue ablation. ${ }^{12}$ The TFL infrared wavelength of $1908 \mathrm{~nm}$ is also four times more strongly absorbed by the water component in tissue than the conventional holmium laser wavelength of $2120 \mathrm{~nm}$, corresponding to an optical penetration depth four times less than for the holmium laser. ${ }^{13}$

We hypothesize that delivery of TFL energy through a small, flexible optical fiber under image guidance using a miniature endoscope may provide a rapid, safe, and effective means of clearing obstructed catheters in patients suffering from hydrocephalus, potentially reducing the need for surgical revision. In this study, preliminary feasibility studies are conducted comparing TFL and holmium laser ablation of tissue phantoms in a ventricular catheter model, ex vivo.

\section{Materials and Methods}

\subsection{Laser Sources and Optical Fiber Delivery Systems}

A 100-W, continuous-wave (CW) TFL (TLR 100-1908, IPG Photonics, Oxford, Massachusetts) with a wavelength of $1908 \mathrm{~nm}$ was electronically modulated with a function generator (Model DS345, Stanford Research Systems, Sunnyvale, California) to produce an energy per pulse of $33 \pm 2 \mathrm{~mJ}$, pulse duration of $500 \mu \mathrm{s}$, and variable pulse rates from 20 to $200 \mathrm{~Hz}$. A 25-mm focal length, antireflection coated, planoconvex lens (LA1560-D, Thorlabs, Newton, New Jersey) was used to focus the $5.5-\mathrm{mm}$-diameter $\left(1 / \mathrm{e}^{2}\right)$ collimated fiber laser beam to a spot diameter of $\sim 25 \mu \mathrm{m}\left(1 / \mathrm{e}^{2}\right)$ for coupling into the optical fiber used in the study.

A low-OH, silica optical fiber (FIPE100140170, Polymicro, Phoenix, Arizona) was cut to a length of $2 \mathrm{~m}$ and consisted of $100-\mu \mathrm{m}$ core, $140-\mu \mathrm{m}$ cladding, and $170-\mu \mathrm{m}$ polyimide jacket. The protective jacket at the distal end of the trunk fiber was stripped using a fiber stripper (Micro-Electronics, Seekonk, Massachusetts), removing a 2- to 5-mm length of cladding for polishing.

For comparison, a clinical Holmium:YAG laser (2.1 XE, Coherent, Santa Clara, California) was operated with a wavelength of $2120 \mathrm{~nm}$, energy per pulse of $325 \pm 25 \mathrm{~mJ}$, pulse duration of $350 \mu \mathrm{s}$, and pulse rate of $10 \mathrm{~Hz}$. The average holmium power of $3.3 \mathrm{~W}$ was similar to the average power for TFL operating at $33 \mathrm{~mJ}$ and $100 \mathrm{~Hz}(3.3 \mathrm{~W})$. A standard $270-\mu \mathrm{m}$ core clinical optical fiber with $450-\mu \mathrm{m}$ jacket (Holmium Lightguide 270D fiber, Olympus Gyrus ACMI) was used for holmium studies. The TFL and holmium fibers used are shown in Fig. 1 for comparison.

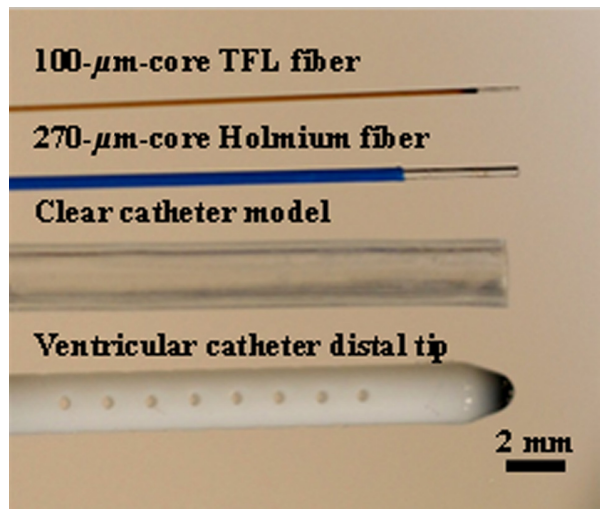

Fig. 1 Physical dimensions of experimental $100-\mu \mathrm{m}$ core $(170-\mu \mathrm{m}$ OD) TFL fiber, standard $270-\mu \mathrm{m}$ core $(450-\mu \mathrm{m}$ OD) holmium fiber, clear silicone tubing (1.3-mm ID/2.3-mm OD) catheter model, and distal tip of commercial ventricular catheter (1.2-mm ID/2.2-mm OD).

Alignment, energy measurement, and optical transmission efficiency tests were performed by measuring the energy per pulse before the proximal end of the fiber and after the distal tip, with a detector (PM150, Coherent, Santa Clara, California) and meter (EPM2000, Molectron, Portland, Oregon).

\subsection{Occluded Catheter Model}

A typical ventricular catheter (41207, Medtronic, Minneapolis, Minnesota) consists of a silicone tube of $1.2 \mathrm{~mm}$ inner diameter (ID) and $2.1 \mathrm{~mm}$ outer diameter (OD) with an array of $500-\mu \mathrm{m}-$ diameter holes around the tube's circumference near the distal tip to allow the inflow of CSF (Fig. 1). Due to the majority of commercial catheters being opaque, similar clear ethyl vinyl acetate tubing with 1.3-mm ID and 2.3-mm OD (1883T4, McMaster-Carr, Elmhurst, Illinois) was used to visualize the occlusions and perform measurements in this study (Fig. 1).

While previous reports have used scallops to simulate choroid plexus, ${ }^{10,11}$ in this study, egg white was chosen as a more consistent model for providing tissue adherence to the catheter wall and occlusion of the catheter lumen. Egg white was injected into 50-mm-length sections of tubing and then baked at $85 \pm 2^{\circ} \mathrm{C}$ for $30 \mathrm{~min}$, so the tissue would stick to the walls, providing an optimal tissue phantom model [Fig. 2(b)]. This process was important for reliable experimental results since undercooked samples not adhering to catheter walls would not hold pressure and, therefore, could be cleared by irrigation or laser vapor bubble pressure too easily within a few seconds and would not represent an accurate model.

A syringe infusion pump (Cole Parmer, Vernon Hills, Illinois), pressure meter (Model 717100 G, Fluke, Everett, Washington), and iris clamp were used to measure a burst pressure of $103.4 \pm 67.3 \mathrm{mmHg}(n=5)$ in optimal tubing samples cut to $15 \mathrm{~mm}$ with an occlusion length of $10 \mathrm{~mm}$. The lumen of the catheter was placed over a cannula attached to the infusion pump. The iris was then closed to seal the catheter onto the cannula. Deionized water was infused at a rate of $100 \mathrm{ml} / \mathrm{h}$, and the pressure was measured with a pressure gauge. The maximum pressure (in $\mathrm{mmHg}$ ) achieved when the vessel seal bursts was then recorded.

\subsection{Tissue Ablation and Thermal Measurements}

The flexible silicone tubing used in the occluded catheter model was straightened by placing it into a $2.5-\mathrm{mm} \mathrm{ID,} \mathrm{3.0-mm} \mathrm{OD}$ 


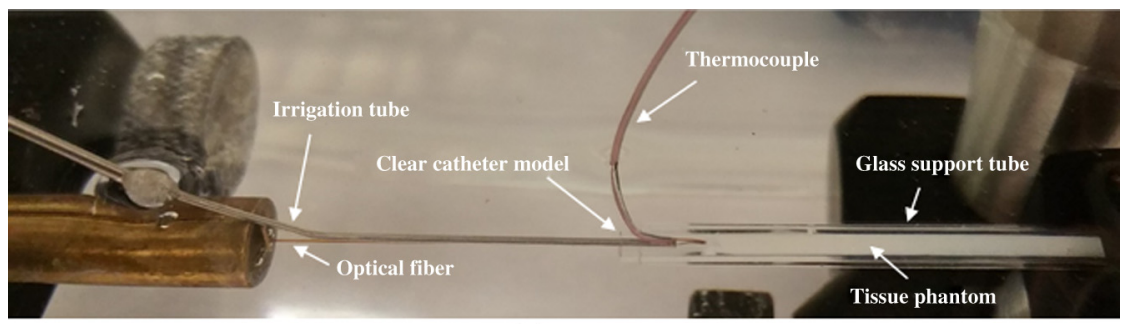

(a)

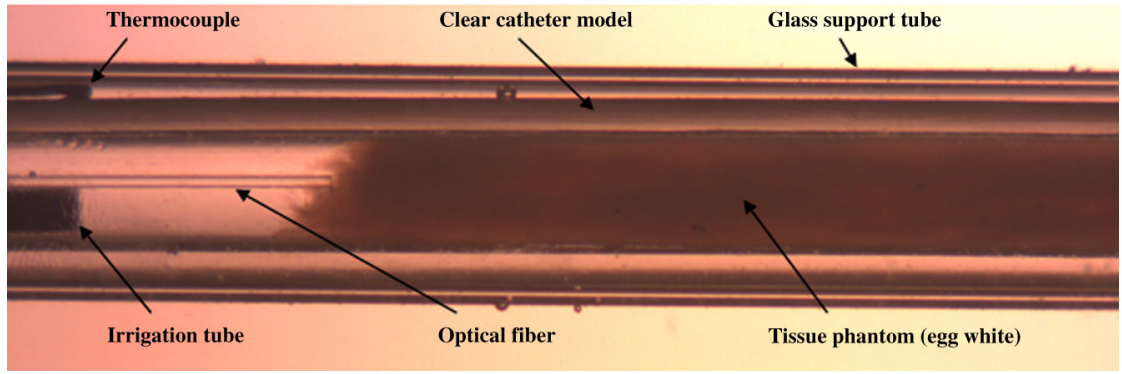

(b)

Fig. 2 (a) Photograph of tissue ablation setup in submerged saline bath. (b) Magnified image of catheter model with 100- $\mu \mathrm{m}$ core (170- $\mu \mathrm{m} \mathrm{OD)} \mathrm{TFL} \mathrm{fiber} \mathrm{and} \mathrm{320-} \mu \mathrm{m}$ ID (370- $\mu \mathrm{m} \mathrm{OD)} \mathrm{irrigation} \mathrm{tube.}$

rigid glass capillary tube (Vitrocom, Mountain Lakes, New Jersey). An insulated, 125- $\mu$ m-diameter thermocouple (Type $\mathrm{T}$, Omega, Norwalk, Connecticut) was used to monitor temperature as a function of time at $500 \mathrm{~ms}$ resolution with a data acquisition system (OM-USB-TC, Omega). The thermocouple was placed between the catheter and glass tube $10 \mathrm{~mm}$ from the cut edge of the tubing and at a 12 o'clock orientation. The tissue setup was then submerged in a transparent saline filled container at room temperature [Fig. 2(a)]. The fiber was secured by a translation stage $50 \mathrm{~mm}$ from the distal tip, to allow for insertion throughout the length of the catheter sample. The fiber was centered within the cross-section of the tube and advanced to be in contact with the tissue, then held fixed during ablation.

Laser ablation time for all studies was kept constant at $10 \mathrm{~s}$. Ablation depth was measured via magnified video from the side [Fig. 2(b)]. If the ablated tissue surface was skewed in the tubing, an average depth was determined based on minimum and maximum distance from the fiber tip. A syringe was periodically inserted into the catheter to irrigate loose debris between ablation tests.

The maximum temperature rise outside the tubing was measured by the thermocouple during ablation. The relative position of the thermocouple to the fiber tip was measured and matched with each recorded temperature. After each ablation test (10 s) when the fiber was advanced into tissue contact again, a new relative thermocouple position measurement was obtained. The tubing was allowed to return to baseline room temperature between tests.

\subsection{High Speed Imaging of Laser-Induced Vapor Bubble}

The catheter model was imaged using a high-speed camera (Fastcam SA5, Photron, Tokyo, Japan) with magnifying lenses $(12 \times$ zoom kit, Navitar, Rochester, New York), providing $10-\mu \mathrm{m}$ spatial resolution and $9.5-\mu \mathrm{s}$ temporal resolution, when operated at 105,000 frames per second. A high power lamp placed behind the container illuminated the fiber and catheter to provide adequate contrast.

\subsection{Laser Damage to Catheter}

For the safety studies, laser damage to the catheter wall was imaged when the fiber was placed directly perpendicular to the catheter wall, and at variable working distances $(0,0.5$, and $1.0 \mathrm{~mm}$ ). Although such an alignment is not practical in a clinical setting due to the tubing geometry, it represented a worst case scenario, perhaps when the fiber encounters the catheter's distal tip or kinks in the catheter. The clear tubing was used for this study to visualize holes from the side and normal to the catheter wall. The TFL was operated at $100 \mathrm{~Hz}$ for $1 \mathrm{~s}$ at various fiber working distances from the catheter wall.

\subsection{Endoscope Guided Procedures}

To demonstrate the feasibility of an endoscope guided laser ablation procedure inside a ventricular catheter, a miniature flexible endoscope (MilliScope II, Advanced Inspection Technologies, Melbourne, Florida) with 0.7-mm OD, 65-deg field of view, $15 \mathrm{~mm}$ bending radius, 3- to 12-mm depth of field, and 10,000 pixels was used [Figs. 3(a) and 3(b)]. A coherent fiber bundle and white light illumination fibers in the endoscope provided live video and images deep within the catheter. The potential advantage of using the TFL with smaller optical fibers inserted into the catheter lumen is shown in the diagrams in Figs. 3(c) and 3(d).

\section{Results}

\subsection{Laser Tissue Ablation Measurements}

A catheter ablation depth of about 1 to $2 \mathrm{~mm}$ was observed for each $10 \mathrm{~s}$ laser activation time, across the range of laser parameters studied (Table 1). Only a slight increase in average ablation depth was observed by increasing the TFL pulse rate. However, due to an increase in vibration at the distal fiber tip during ablation, higher laser pulse rates also resulted in higher catheter failure rates, as judged by the number of catheter sidewall damage events (Table 1). It should be noted that proper guidance and 


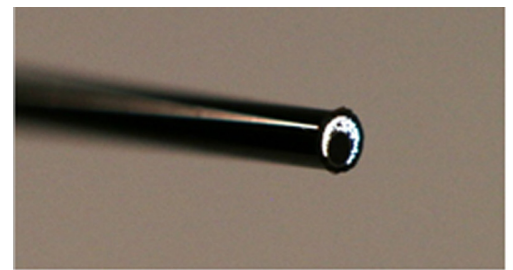

(a)

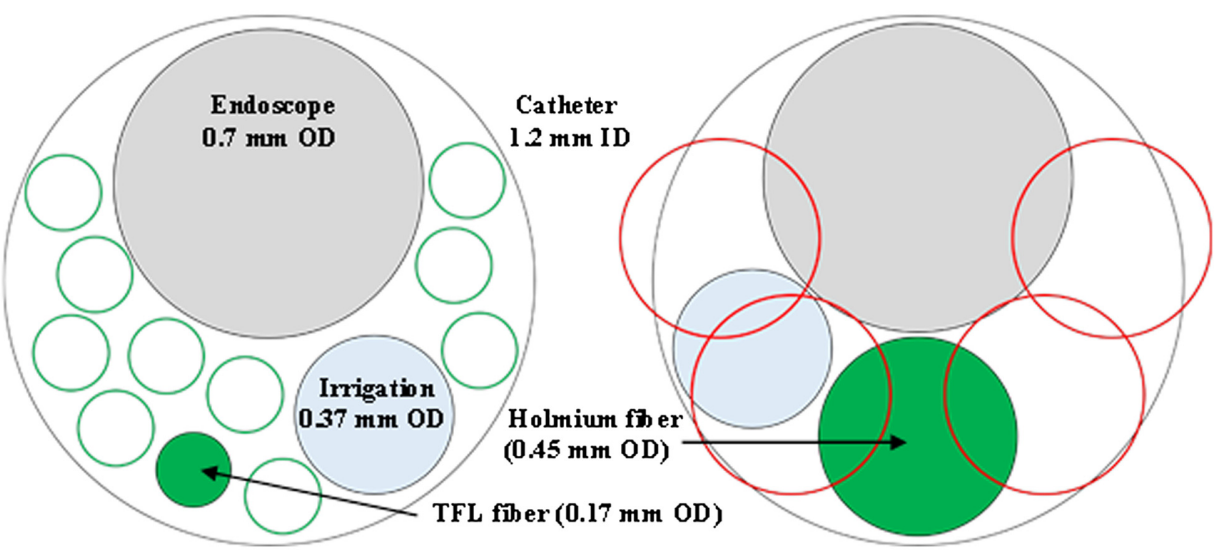

(c)

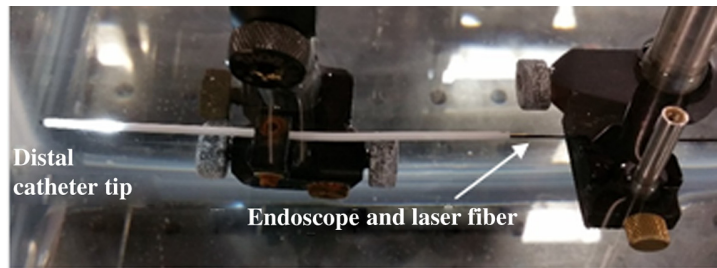

(b)

(d)

Fig. 3 (a) Photograph of distal tip of 0.7-mm OD miniature endoscope, showing illumination fibers. The dark region is coherent fiber bundle for imaging. (b) Photograph of submerged endoscope setup in commercial ventricular catheter. (c, d) Cross-sectional diagram of catheter lumen with scale endoscope, irrigation tube, and (c) TFL fiber, and (d) holmium fiber. Also illustrated are alternative positions of the laser fiber. Due to the larger outer diameter of the holmium fiber (red circles), additional positions are impossible.

Table 1 Comparison of holmium:YAG and TFL for clearance of occluded catheters. An ablation time of $10 \mathrm{~s}$ was used. Damage to the catheter sidewall was noted as a failure in terms of safety rates. ${ }^{a}$

\begin{tabular}{|c|c|c|c|c|c|c|}
\hline Laser & Energy (mJ) & Pulse rate $(\mathrm{Hz})$ & Ablation depth $(\mathrm{mm})$ & $N$ (success) & $N($ sidewall) & Safety rate $(\%)$ \\
\hline TFL & 33 & 20 & $0.8 \pm 0.5$ & 20 & 0 & 100 \\
\hline TFL & 33 & 50 & $1.5 \pm 1.0$ & 37 & 1 & 97 \\
\hline TFL & 33 & 100 & $1.6 \pm 0.9$ & 42 & 9 & 82 \\
\hline TFL & 33 & 200 & $2.0 \pm 0.8$ & 23 & 17 & 58 \\
\hline Ho:YAG & 325 & 10 & $1.3 \pm 0.5$ & 25 & 0 & 100 \\
\hline
\end{tabular}

aHigher sidewall damage events were observed as TFL pulse rate increased due to greater distal fiber tip vibration.

${ }^{\mathrm{b}} \mathrm{A}$ student's $t$-test was used for statistical comparison of ablation depth to TFL at $100 \mathrm{~Hz}$, yielding $p<0.01$ for TFL at $20 \mathrm{~Hz}, p=0.44$ for TFL at $50 \mathrm{~Hz}, p=0.02$ for TFL at $200 \mathrm{~Hz}$, and $p=0.09$ for holmium laser.

handling of the distal fiber tip through an endoscope could potentially solve this problem. A comparison of TFL (33 mJ and $100 \mathrm{~Hz}$ ) and holmium $(325 \mathrm{~mJ}$ and $10 \mathrm{~Hz})$ parameters for similar average powers $(3.3 \mathrm{~W})$ yielded similar ablation depths of $1.6 \pm 0.9$ and $1.3 \pm 0.5 \mathrm{~mm}$, respectively $(p=0.09)$. There was also no statistical difference in ablation depths between TFL at 50 and $100 \mathrm{~Hz}(p=0.44)$. Taking into consideration both higher distal fiber tip vibration at $100 \mathrm{~Hz}$ translating into higher sidewall damage or failure rates and no statistical difference in ablation depths, TFL operation at $50 \mathrm{~Hz}$ appears to be a better choice of parameters.

For each trial, the fiber tip was placed in contact with the occlusion [Fig. 4(a)]. After $10 \mathrm{~s}$ laser exposure, debris would either be ablated or dislodged from the catheter wall [Fig. 4(b)]. An irrigation tube [Fig. 2(b)] was then extended to the site to flush away debris toward the proximal end of the catheter, leaving only the remaining tissue adhered to the wall [Fig. 4(c)]. For the first trial of each sample, the opaque region of the sample was used for ablation depth measurements. Figure 4(a) shows a typical condition after irrigation of a sample's first ablation trial. Once the tissue in the sample was exalted, and the lumen essentially free flowing, typically after about 10 trials, the tube was removed and replaced with a fresh sample. While tissue commonly would be observed to stick to the fiber tip, possibly due to fiber tip heating, studies were continued, and distal fiber tip degradation was not observed after soft tissue ablation. 


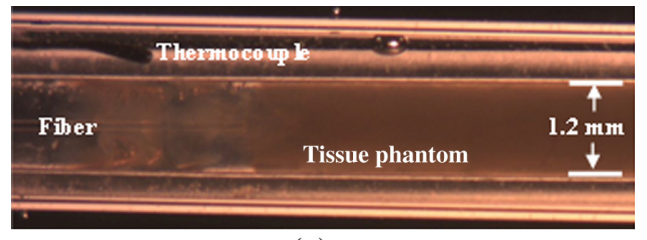

(a)

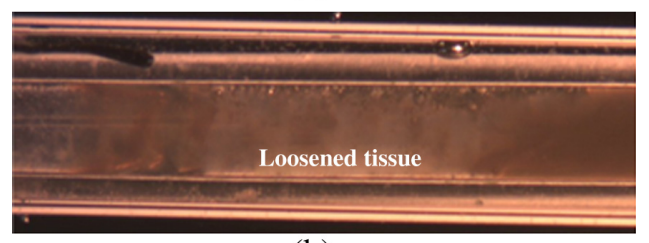

(b)

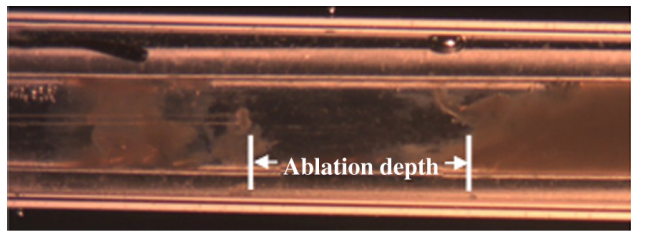

(c)

Fig. 4 Tissue ablation trial showing (a) before laser exposure, (b) after TFL exposure at $50 \mathrm{~Hz}$ for $10 \mathrm{~s}$, and (c) after saline irrigation of dislodged debris. An average ablation depth measurement was then obtained for the trial.

\subsection{Thermal Measurements}

During each ablation trial, the temperature rise of the outer catheter wall was recorded with a thermocouple. A dependence on the thermocouple's position relative to the fiber tip was observed (Fig. 5). The peak temperature rise on the catheter wall occurred at the 2-mm location in front of the fiber tip and, on average, increased with pulse rate, with maximum increases of about $10^{\circ} \mathrm{C}$ and $20^{\circ} \mathrm{C}$ for 50 and $200 \mathrm{~Hz}$, respectively. Temperature tended to decrease as measurements were acquired beyond the ablation site, with temperatures decaying more rapidly toward the distal end with tissue and gradually toward the proximal end, which contained laser-heated saline. If the fiber came in prolonged contact with the catheter sidewall at a glancing angle, a sharp increase in temperature was typically observed with occasional swelling of the catheter wall. The largest temperature rise $\left(35^{\circ} \mathrm{C}\right)$ occurred during the first trial of a sample where the fiber was only inserted $1 \mathrm{~mm}$ into the sample tube and it was later determined that the fiber was mistakenly irradiating the catheter sidewall at an angle. Charring occurred and the trial lasted for $10 \mathrm{~s}$ to observe maximum temperature rise. Baseline room temperature for all tests measured $22.5 \pm 0.5^{\circ} \mathrm{C}$ $(n=174)$.

\subsection{High-Speed Imaging of Laser-Induced Vapor Bubble}

The laser-induced vapor bubble created in contact with tissue directed the majority of expansion toward the proximal end of the catheter, and the bubble rebounded back toward the tissue with a center of collapse near the fiber tip (Fig. 6). This enabled direct laser tissue heating during the pulse, resulting in ablation; additionally, the subsequent collapse of the bubble provided mechanical disruption to the tissue as well. The rapid bubble expansion also slightly compressed the tissue due to elasticity. Superheated tissue maintained a thin vapor layer, occasionally increasing bubble size between pulses. A reduced expansion toward the proximal end was observed when clamping the catheter closed during ablation, due to the inability of the saline to be displaced in that direction, presumably increasing vapor pressure and tissue heating.

\subsection{Thulium Fiber Laser Damage to Ventricular Catheter Wall}

Direct laser irradiation of the catheter wall for $1 \mathrm{~s}$ at $33 \mathrm{~mJ}$ and $100 \mathrm{~Hz}$ showed irreversible swelling and pitting (Fig. 7). Closer fiber working distances to the catheter wall increased the damage. Fiber to wall working distances of 0 (contact mode), 0.5 , and $1 \mathrm{~mm}$ produced a hole, shallow crater, and swelling, respectively.

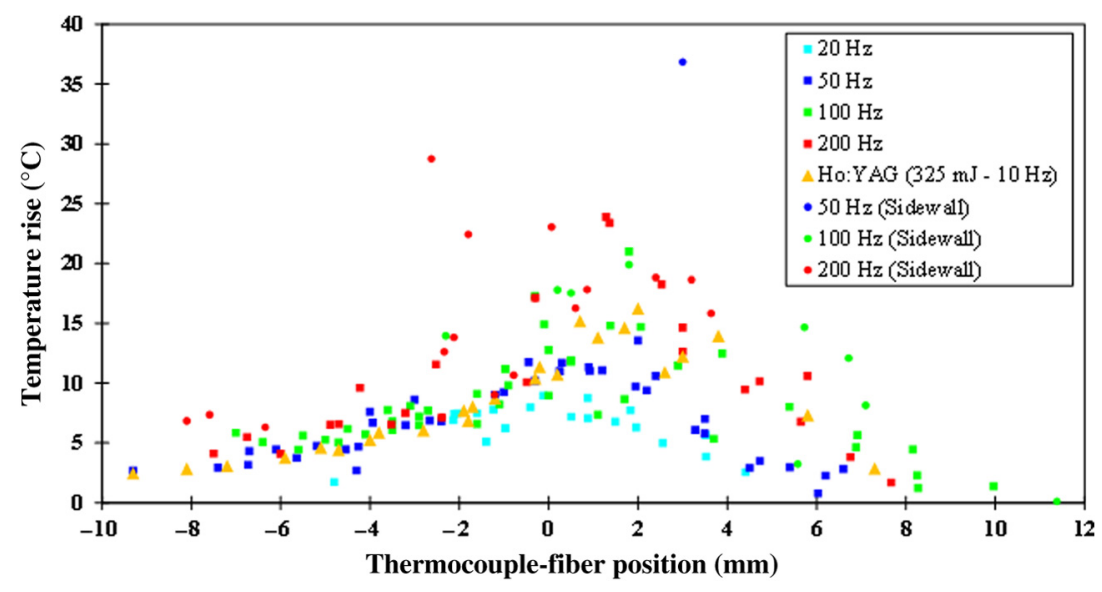

Fig. 5 Temperature rise of silicone catheter tubing relative to optical fiber position. All data points are for $10 \mathrm{~s}$ exposure time in contact with the tissue phantom for either TFL at $33 \mathrm{~mJ}$ and 20 to $200 \mathrm{~Hz}$ or holmium:YAG laser at $325 \mathrm{~mJ}$ and $10 \mathrm{~Hz}$. Data points (circles) are shown separately for events in which accidental TFL irradiation of the catheter sidewall were observed, due to excessive vibration of the distal fiber tip. Such adverse events may potentially be prevented by stabilization of the distal fiber tip within the working channel of an endoscope during the procedure. 


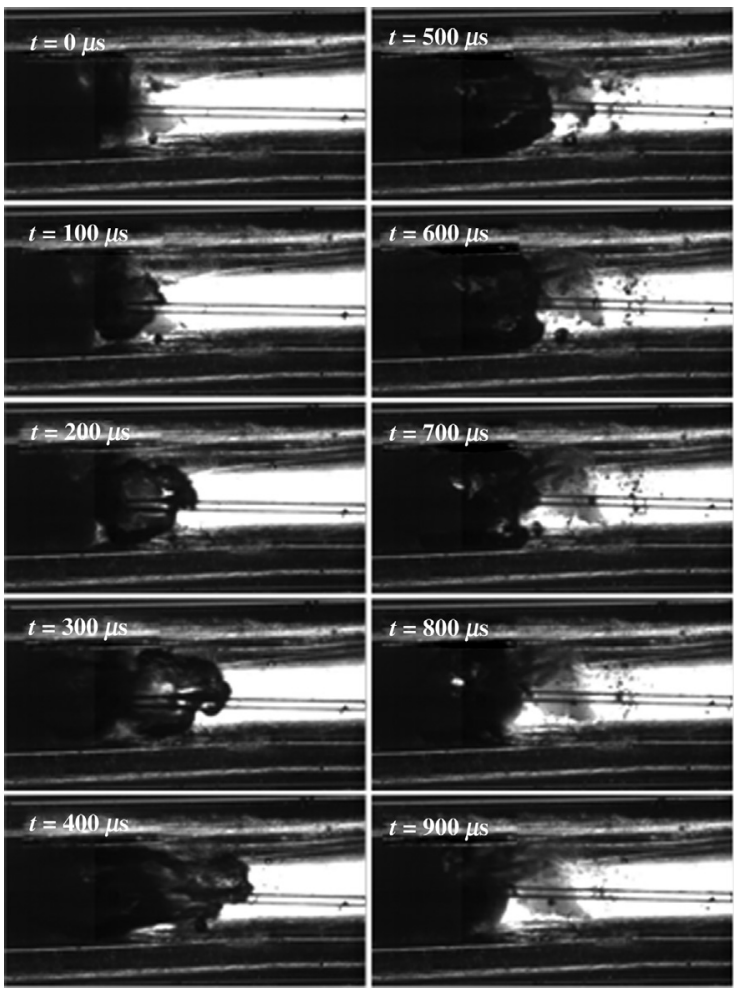

Fig. 6 High-speed camera images of TFL-induced vapor bubble expansion and collapse during a single $500-\mu \mathrm{s}$-long laser pulse. The TFL $100-\mu \mathrm{m}$ core fiber was in contact with the tissue phantom in the catheter model. The proximal end of the catheter was open inside the saline container.

\subsection{Endoscope Assisted Surgery}

In Fig. 8, images from a 0.7-mm OD, 10,000 pixel miniature endoscope are shown, simulating an image guided laser ablation procedure. The distal tip of the $100-\mu \mathrm{m}$ core (170- $\mu \mathrm{m}$ OD) optical fiber, $370-\mu \mathrm{m}$ OD irrigation tube, the clear catheter wall, and the egg white tissue phantom can be differentiated.

\section{Discussion}

Several experimental and clinical laser applications for treatment of hydrocephalus have been previously reported. These include the pulsed holmium:YAG laser for fiber optic based clearance of occluded shunts, ${ }^{10,11}$ as well as continuous-wave neodymium:YAG, thulium:YAG, and diode lasers for endoscopic third ventriculostomy, using either bare optical fibers or carbon ball tip optical fibers for precise tissue ablation. ${ }^{14-21}$

The primary advantage of the TFL is its near single mode spatial beam profile, which enables coupling of high laser power into ultrasmall (50 and $100 \mu \mathrm{m}$ core) optical fibers. This property frees up valuable cross-sectional space within the typical 1.2-mm ID lumen of the ventricular catheter for simultaneous use of a miniature endoscope for image guidance and saline irrigation tube for visibility and safety during the procedure. The conventional holmium laser, by comparison, is limited by its multimode spatial beam profile for use with larger fibers (270- $\mu \mathrm{m}$ core and 450- $\mu \mathrm{m}$ OD). Otherwise, tissue ablation rates between TFL and holmium lasers in this study were comparable and similar to previous experimental holmium laser studies. ${ }^{10,11}$

Laser clearance of obstructed catheters appeared to be due primarily to a photomechanical mechanism represented by the rapid expansion and contraction of the laser-induced vapor bubble at the distal fiber tip when immersed in the saline-filled catheter tube, rather than a photothermal tissue ablation mechanism. It is well understood that when IR laser energy is delivered in pulsed mode through a fiber immersed in a fluid medium, a rapidly expanding and contracting vapor bubble is created at the fiber tip during the laser pulse duration. This laser-induced vapor bubble not only allows the laser energy to propagate through a highly absorbing medium (by effectively "parting the water," known as "Moses effect") but also produces pressure transients that in turn lead to a mechanical component to tissue ablation or fragmentation. The resulting mechanical wave can be exploited to provide a safe, nonthermal method for tissue clearance from the catheter wall. The conventional holmium surgical laser, typically operated at high pulse energy and low pulse

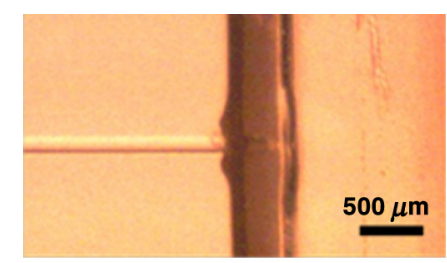

(a)

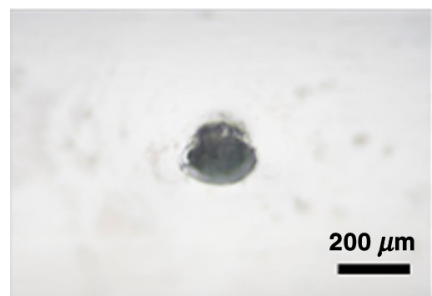

(d)

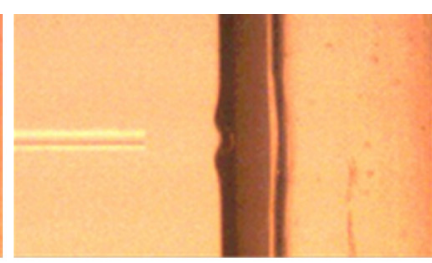

(b)

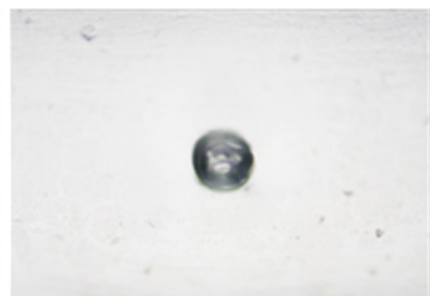

(e)

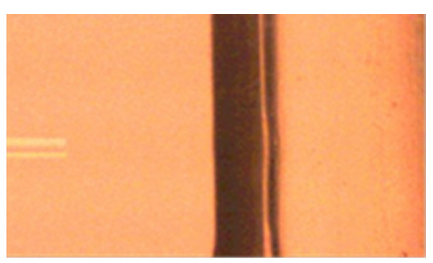

(c)

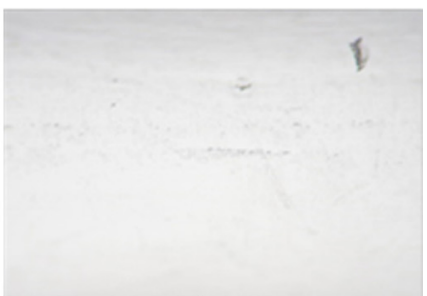

(f)

Fig. 7 TFL with 100- $\mu$ m core fiber placed perpendicular to the silicone catheter wall and operated at $33 \mathrm{~mJ}$ and $100 \mathrm{~Hz}$ for $1 \mathrm{~s}$. Side view (top) and normal incident view (bottom) after laser exposure at different fiber to wall working distances of $(a, d) 0 \mathrm{~mm},(b, e) 0.5 \mathrm{~mm}$, and (c, f) $1 \mathrm{~mm}$. These three working distances of $0,0.5$, and $1 \mathrm{~mm}$ produced holes, shallow craters, and swelling, respectively. 


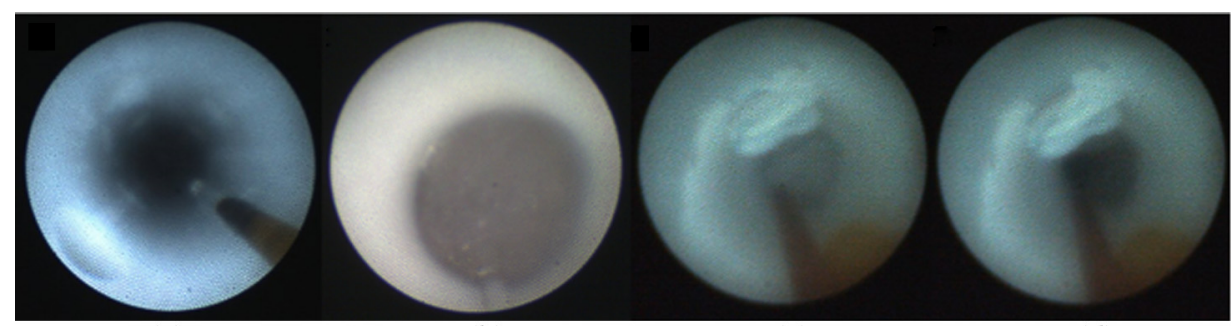

(a)

(b)

(c)

(d)

Fig. 8 Images from 0.7-mm OD, 10,000 pixel endoscope, simulating an image-guided laser ablation procedure. (a) Clear catheter near distal tip with CSF drainage holes and side-by-side 100- $\mu \mathrm{m}$ core fiber. (b) Clear catheter at distal tip with radiopaque plug. Egg white sample prepared in opaque white commercial catheter with side-by-side $100-\mu \mathrm{m}$ core fiber and $370-\mu \mathrm{m}$ OD irrigation tube (c) before and (d) after ablation.

rates, produces large (4 to $5 \mathrm{~mm}$ length) vapor bubbles. The TFL when operated at low pulse energies and high pulse rates produces smaller ( 1 to $2 \mathrm{~mm}$ length) bubbles. ${ }^{22}$ Future studies will explore whether this difference can be exploited to enable the TFL to provide a more precise photomechanical effect, not only to reduce the probability of collateral damage to the catheter wall and surrounding tissues but also potentially to clear some of the small, $500-\mu \mathrm{m}$ ID, circumferential holes in the ventricular catheter in addition to the lumen. Clearance of only a few of the 32 holes may be sufficient for normal drainage of the ventricular catheter.

Vibration of the distal fiber tip during TFL studies at high pulse rates $(100$ and $200 \mathrm{~Hz})$ resulted in higher failure rates (as defined by accidental laser-induced thermal damage to the catheter sidewall) than for the holmium laser. However, this problem may be eliminated by future use of a miniature endoscope with an integrated working channel for fiber insertion, centering, and stability, which was not available during these preliminary studies.

One challenge for laser clearance of occluded shunts is the lack of a universal shunt design for ventricular catheters and valves used in the treatment of hydrocephalus. Future studies will therefore investigate the feasibility of inserting optical fibers through a variety of ventricular catheter designs and valve configurations.

Furthermore, studies will also investigate a more realistic tissue model, based on ventricular catheters explanted during surgical revisions. Our egg white tissue phantom was initially chosen due to its ability to reliably adhere to the catheter wall and provide consistent occlusion of the catheter lumen. Additionally, the optical, thermal, and damage parameters for coagulated egg white are readily available in the literature. ${ }^{23}$ Previous studies have used sea scallops to represent choroid plexus tissue during experimental studies; ${ }^{10,11}$ however, we were unable to achieve sufficient tube wall adherence with a scallop model similar to that observed with coagulated egg white.

The TFL laser may also be explored for more precise and safer ablation of brain tissue adhering to the outside surface of ventricular catheters during surgical revision procedures, rather than the current approach of using monopolar electrocautery, which produces greater thermal spread.

\section{Conclusions}

Holmium:YAG and TFLs were compared for tissue ablation in an ex vivo ventricular catheter tissue phantom model, providing similar ablation rates. With further development, simultaneous application of the TFL with small fibers, miniature endoscope for image guidance, and saline irrigation tube for removal of tissue debris, may provide a safe and efficient minimally invasive method of clearing occluded ventricular catheters for treatment of hydrocephalus.

\section{Disclosures}

None of the authors have a financial interest in this manuscript.

\section{Acknowledgments}

This research was supported by a seed grant from the Charlotte Research Institute. David Gonzalez was supported by a summer research grant from the Charlotte Research Scholars program at UNC Charlotte.

\section{References}

1. Hydrocephalus Association, http://www.hydroassoc.org/about-us/ newsroom/facts-and-stats-2/.

2. G. K. Reddy, P. Bollam, and G. Caldito, "Long-term outcomes of ventriculoperitoneal shunt surgery in patients with hydrocephalus," World Neurosurg. 81(2), 404-410 (2014).

3. J. M. Drake and J. Kestle, "Rationale and methodology of the multicenter pediatric cerebrospinal fluid shunt design trial," Child's Nerv. Syst. 12(8), 434-447 (1996).

4. R. D. Brownlee, O. N. Dold, and S. T. Myles, "Intraventricular hemorrhage complicating ventricular catheter revision: incidence and effect on shunt survival," Pediatr. Neurosurg. 22(6), 315-320 (1995).

5. R. V. Patwardhan and A. Nanda, "Implanted ventricular shunts in the United States: the billion-dollar-a-year cost of hydrocephalus treatment," Neurosurgery 56(1), 139-145 (2005).

6. J. V. Pattisapu et al., "Percutaneous endoscopic recanalization of the catheter: a new technique of proximal shunt revision," Neurosurgery 45(6), 1361-1366 (1999).

7. H. J. Ginsberg, J. M. Drake, and R. S. C. Cobbold, "Unblocking cerebrospinal fluid shunts using low frequency ultrasonic cavitation," in Proc. IEEE Ultrasonics Symp., pp. 1381-1384 (2001).

8. H. J. Ginsberg et al., "Recanalization of obstructed cerebrospinal fluid ventricular catheters using ultrasonic cavitation," Neurosurgery 59, 403-412 (2006).

9. R. Fox and J. Norton, "A novel device for the clearance and prevention of blockages within biomedical catheters," Med. Eng. Phys. 36(11), 1526-1529 (2014)

10. W. A. Christens-Barry, M. Guarnieri, and B. S. Carson, "Laser restoration of flow in occluded ventricular shunts for pediatric neurosurgery," Proc. SPIE 3193, 140 (1998).

11. W. A. Christens-Barry, M. Guarnieri, and B. S. Carson, "Fiberoptic delivery of laser energy to remove occlusions from ventricular shunts: technical report," Neurosurgery 44(2), 345-349 (1999).

12. R. L. Blackmon et al., "Thulium fiber laser ablation of kidney stones using a 50- $\mu \mathrm{m}$-core silica optical fiber," Opt. Eng. 54(1), 011004 (2015). 
13. R. L. Blackmon, P. B. Irby, and N. M. Fried, "Comparison of holmium: YAG and thulium fiber laser lithotripsy: ablation thresholds, ablation rates, and retropulsion effects," J. Biomed. Opt. 16(7), 071403 (2011).

14. R. D. Bucholz and T. Pittman, "Endoscopic coagulation of the choroid plexus using the Nd:YAG laser: initial experience and proposal for management," Neurosurgery 28(3), 421-427 (1991).

15. W. P. Vandertop, R. M. Verdaasdonk, and C. F. van Swol, "Laserassisted neuroendoscopy using a neodymium-yttrium aluminum garnet or diode contact laser with pretreated fiber tips," J. Neurosurg. 88(1), 82-92 (1998).

16. P. W. A. Williams et al., "Contact laser-assisted neuroendoscopy can be performed safely by using pretreated 'black' fibre tips: experimental data," Lasers Surg. Med. 28(4), 324-329 (2001).

17. B. C. Devaux et al., "Laser-assisted endoscopic third ventriculostomy for obstructive hydrocephalus: technique and results in a series of 40 consecutive cases," Lasers Surg. Med. 34(5), 368-378 (2004).

18. H. C. Ludwig et al., "First experiences with a 2.0-micron near infrared laser system for neuroendoscopy," Neurosurg. Rev. 30(3), 195-201 (2007).

19. J. van Beijnum et al., "Laser-assisted endoscopic third ventriculostomy: long-term results in a series of 202 patients," Neurosurgery 62(2), 437-444 (2008).

20. F. H. Ebner et al., "Efficacy and versatility of the 2-micron continuouswave laser in neuroendoscopic procedures," Acta Neurochir. Suppl. 113, 143-147 (2012).
21. R. C. Reis et al., "Application of a 980-nanometer diode laser in neuroendoscopy: a case series," J. Neurosurg. 124(2), 368-374 (2016).

22. L. A. Hardy et al., "Analysis of thulium fiber laser induced vapor bubbles for ablation of kidney stones," J. Biophotonics (In Press).

23. Y. Yang, A. J. Welch, and H. G. Rylander, "Rate process parameters of albumen," Lasers Surg. Med. 11(2), 188-190 (1991).

Thomas C. Hutchens, $\mathrm{PhD}$, is a postdoctoral fellow in the Department of Physics and Optical Science at the University of North Carolina at Charlotte.

David A. Gonzalez is an undergraduate physics major at the University of North Carolina at Charlotte.

Luke A. Hardy is a PhD student in the Optical Science and Engineering Program at the University of North Carolina at Charlotte.

C. Scott McLanahan, MD, is the Director of the Adult Hydrocephalus Specialty Clinic at Carolinas Medical Center in Charlotte, North Carolina, USA.

Nathaniel M. Fried, $\mathrm{PhD}$, is a professor in the Department of Physics and Optical Science at the University of North Carolina at Charlotte. His research interests include therapeutic and diagnostic applications of lasers in medicine. 